\title{
Event-triggered robust guaranteed cost control for two-dimensional nonlinear discrete-time systems
}

\author{
WANG Sen, BU Xuhui", and LIANG Jiaqi \\ School of Electrical Engineering and Automation, Henan Polytechnic University, Jiaozuo 454000, China
}

\begin{abstract}
An event-triggered scheme is proposed to solve the problems of robust guaranteed cost control for a class of two-dimensional (2-D) discrete-time systems. Firstly, an eventtriggered scheme is proposed for 2-D discrete-time systems with parameter uncertainties and sector nonlinearities. Then, according to the Lyapunov functional method, the sufficient conditions for the existence of event-triggered robust guaranteed cost controller for 2-D discrete-time systems with parameter uncertainties and sector nonlinearities are given. Furthermore, based on the sufficient conditions and the linear matrix inequality (LMI) technique, the problem of designing event-triggered robust guaranteed cost controller is transformed into a feasible solution problem of LMI. Finally, a numerical example is given to demonstrate that, under the proposed event-triggered robust guaranteed cost control, the closed-loop system is asymptotically stable and fewer communication resources are occupied.
\end{abstract}

Keywords: event-triggered, robust guaranteed cost control, twodimensional (2-D) nonlinear system, networked control system.

DOI: $10.21629 /$ JSEE.2019.06.18

\section{Introduction}

The two-dimensional (2-D) systems have attracted a lot of interest and are widely used in process control, image processing, multi-dimensional signal filtering and other fields [1-3]. The 2-D system has the typical characteristics of the multi-dimensional system, and its information and energy are transmitted in two directions. Therefore, the research on the stability and stabilization of 2-D systems has practical significance. As stated in [2], the state-space models used to describe 2-D systems are mainly the Attasi model, the Fornasini-Marchesini (FM) model and the Roesser model. However, the Roesser model, which is the

\footnotetext{
Manuscript received November 12, 2018.

*Corresponding author.

This work was supported by the National Natural Science Foundation of China (61573129; U1804147), the Innovative Scientists and Technicians Team of Henan Provincial High Education (20IRTSTHN019), and the Innovative Scientists and Technicians Team of Henan Polytechnic University (T2019-2; T2017-1).
}

most widely used to describe two-dimensional systems, is simple in structure and can be transformed from other models. Hence the 2-D system studied in this paper is also described by the Roesser model.

The problem of nonlinearity has always been an important part of the control system. If the nonlinear problem cannot be solved effectively, it will pose a great threat to system performance and stability. At present, some researches have been done on 2-D nonlinear systems: in [4,5], for the 2-D discrete systems with sector nonlinearity, by constructing the Lyapunov function system stability and using the linear matrix inequality (LMI) method, the design methods of state feedback controller and $H_{\infty}$ controller were studied. In [6,7], the stability and robust controller of 2-D discrete systems which have sector nonlinearity and intermittent measurement were studied. In [8], the stability problem of 2-D discrete systems with statedelayed and saturation nonlinearities was investigated. In [9], considering a class of generalized Lipschitz nonlinearities, the problem of robust $H_{\infty}$ control for uncertain 2-D discrete state-delayed systems was investigated. It is worth mentioning that the research on a class of sector nonlinear systems has received more and more attention [10].

On the other hand, due to the model uncertainty, it is difficult for the system model to describe the actual control system accurately. Therefore, the design of the robust controller becomes a problem that cannot be ignored. Designing a guaranteed cost controller is a good solution to the problem of designing a robust controller. The guaranteed cost controller makes the system robustly asymptotically stable and the upper bound of the cost function exist. The guaranteed cost control of uncertain 2-D discrete systems has received much attention [11-14]. In [11], a robust guaranteed cost state feedback controller for 2-D uncertain discrete systems by the LMI method was designed. In [14], the robust guaranteed cost control problem for 2-D uncertain systems with time-delay was studied. These papers are mainly for uncertain 2-D linear discrete-time sys- 
tems, and the research results on uncertain 2-D nonlinear discrete robust guaranteed cost control are still few.

However, all the above-mentioned guaranteed cost controllers for 2-D systems use time-triggered scheme (or periodic sampling), that is, the controller design requires system data for each sampling instant. From the perspective of resource utilization, when the sampling period of the system is small, the time triggering mechanism generates a large number of redundant sampling signals, causing communication resources to be congested, resulting in waste of computing resources and battery equipment. The event-triggered scheme refers to whether the control task is executed by a predetermined event-triggered condition, not according to the time situation. Therefore, eventtriggered control has attracted the attention of considerable research interest. Among them $[15,16]$ first verified the event-triggered control method can effectively reduce the number of control task execution, showing the advantages based on event-triggered control. Today, there are two main design methods for event-triggered control schemes in existing research results. One is to first design control gain and then design event-triggered condition [17-19]. The other is a method called co-design method that generates control gains while designing event-triggered conditions [20-24]. An event-triggered guaranteed cost controller for 1-D systems was designed in [21] using the co-design method.

In this paper, considering results in [4] and [21], which design event-triggered guaranteed cost controller for 1-D system and analysis stability and stabilisation for a class of 2-D nonlinear discrete systems, the problem of eventtriggered guaranteed cost control for uncertain 2-D nonlinear systems is studied in the network environment. The system we study is a class of 2-D discrete systems with norm-bounded parameter uncertainties and sector nonlinearity. For this system, we propose the event-triggered condition. Then, we design the event-triggered guaranteed cost controller of the system. Under the action of the controller, the closed-loop system is robustly asymptotically stable, and the cost function of the system has the upper bound.

This paper has five parts. The first part is the introduction, that is the current section. The second part is the problem description, which is proposed in Section 2. The third part are the main results of this paper, which are presented in Section 3. The fourth part is the numerical simulation and the result analysis, which show the advantage of the proposed approaches in Section 4 and the fifth part is the conclusion showed in Section 5.

\section{Problem formulation}

Consider a class of 2-D uncertain discrete systems with sector nonlinearity described by the Roesser model:

$$
\left\{\begin{array}{c}
{\left[\begin{array}{c}
\boldsymbol{x}^{h}(i+1, j) \\
\boldsymbol{x}^{v}(i, j+1)
\end{array}\right]=(\boldsymbol{A}+\Delta \boldsymbol{A})\left[\begin{array}{l}
\boldsymbol{x}^{h}(i, j) \\
\boldsymbol{x}^{v}(i, j)
\end{array}\right]+} \\
\left(\boldsymbol{B}_{1}+\Delta \boldsymbol{B}_{1}\right) \boldsymbol{u}(i, j)+(\boldsymbol{B}+\Delta \boldsymbol{B}) \psi(\boldsymbol{v}) \\
\boldsymbol{v}=\boldsymbol{C}\left[\begin{array}{l}
\boldsymbol{x}^{h}(i, j) \\
\boldsymbol{x}^{v}(i, j)
\end{array}\right]
\end{array}\right.
$$

where $\boldsymbol{x}^{h}(i, j) \in \mathbf{R}^{n_{1}}, \boldsymbol{x}^{v}(i, j) \in \mathbf{R}^{n_{2}}$ represent a state vector in the horizontal direction and a state vector in the vertical direction respectively; $\boldsymbol{u}(i, j) \in \mathbf{R}^{m}$ denotes the control input; $\boldsymbol{A}, \boldsymbol{B}, \boldsymbol{B}_{1}, \boldsymbol{C}$ are known constant matrices with the appropriate dimensions. $\Delta \boldsymbol{A}, \Delta \boldsymbol{B}, \Delta \boldsymbol{B}_{1}$ are unknown matrices, representing uncertainties in system, which has the following structure:

$$
\left\{\begin{array}{l}
\Delta \boldsymbol{A}=\boldsymbol{D} \boldsymbol{F}(i, j) \boldsymbol{E}_{1} \\
\Delta \boldsymbol{B}_{1}=\boldsymbol{D} \boldsymbol{F}(i, j) \boldsymbol{E}_{2} \\
\Delta \boldsymbol{B}=\boldsymbol{D} \boldsymbol{F}(i, j) \boldsymbol{E}_{3}
\end{array}\right.
$$

where $\boldsymbol{D}, \boldsymbol{E}_{1}, \boldsymbol{E}_{2}, \boldsymbol{E}_{3}$ are known constant matrices of appropriate dimensions, $\boldsymbol{F}(i, j)$ represents uncertain parameter perturbation of the system, which is the unknown matrix and satisfies $\boldsymbol{F}^{\mathrm{T}} \boldsymbol{F} \leqslant \boldsymbol{I}$.

The function $\psi(\boldsymbol{v})$ denotes the standard vector-valued sector nonlinearity defined as follows.

Definition 1 [10] A memory less vector nonlinear function $\psi: \mathbf{R}^{n} \rightarrow \mathbf{R}^{n}$ is said to satisfy a sector condition if

$$
\left(\psi(\boldsymbol{v})-\boldsymbol{K}_{1} \boldsymbol{v}\right)^{\mathrm{T}}\left(\psi(\boldsymbol{v})-\boldsymbol{K}_{2} \boldsymbol{v}\right) \leqslant 0
$$

for some diagonal real matrices $\boldsymbol{K}_{1}, \boldsymbol{K}_{2} \in \mathbf{R}^{n \times n}$, where $\boldsymbol{K}=\boldsymbol{K}_{2}-\boldsymbol{K}_{1}$ is a positive definite symmetric matrix. Then $\psi$ belongs to the sector $\left[\boldsymbol{K}_{1}, \boldsymbol{K}_{2}\right]$ and can be denoted by $\psi \in\left[\boldsymbol{K}_{1}, \boldsymbol{K}_{2}\right]$.

The boundary conditions of the system satisfy the following assumptions.

Assumption 1 [12] Assumes that system (1) has a finite initial set of conditions, there are two positive integers $m$ and $n$, such that

$$
\left\{\begin{array}{l}
\boldsymbol{x}^{h}(0, j)=\mathbf{0}, \quad j \geqslant n \\
\boldsymbol{x}^{v}(i, 0)=\mathbf{0}, \quad i \geqslant m
\end{array}\right.
$$

and the initial conditions of the system are arbitrary, but belong to the set $\boldsymbol{S}$ which has the following form:

$$
\begin{gathered}
\boldsymbol{S}=\left\{\boldsymbol{x}^{h}(0, j), \boldsymbol{x}^{v}(i, 0): \boldsymbol{x}^{h}(0, j)=\boldsymbol{L} \boldsymbol{N}_{1},\right. \\
\left.\boldsymbol{x}^{v}(i, 0)=\boldsymbol{L} \boldsymbol{N}_{2}, \boldsymbol{N}_{t}^{\mathrm{T}} \boldsymbol{N}_{t}<1(t=1,2)\right\}
\end{gathered}
$$

where $\boldsymbol{L}$ is a known matrix.

Unlike the traditional point-to-point control system, the system (1) is a network control system, and data between the sensors and the controller is transmitted through the communication network. In general, the communication resources of the communication network are limited. In order to reduce the unnecessary data transmission while ensuring system performance and saving communication 
resources, an event-triggered generator is set between the sensor and the controller, and its system structure diagram is shown in Fig. 1. According to the designed eventtriggered condition, it is judged whether the data of the sensor at each sampling moment is transmitted to the controller. The current sensor measurement is transmitted to the controller only when the event-triggered condition is met, then the control input signal is updated. Otherwise, the control input signal is held by the zero-order holder $(\mathrm{ZOH})$.

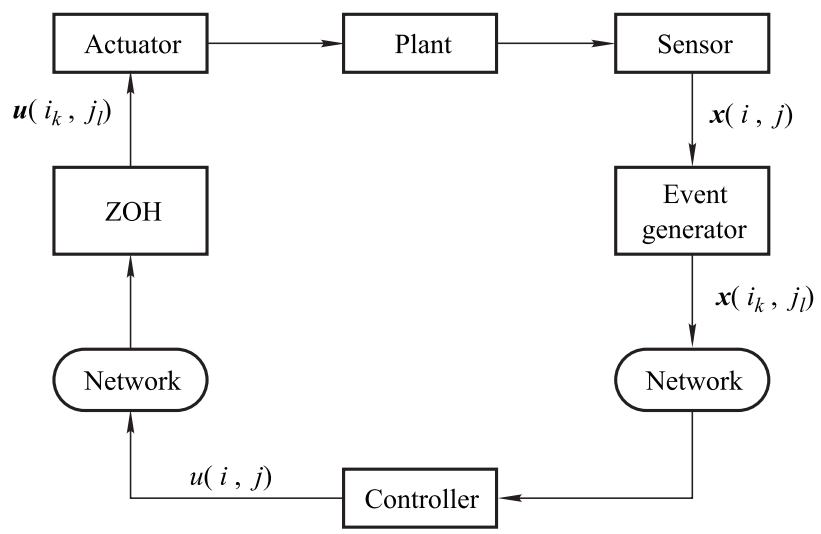

Fig. 1 Structure of event-triggered system

In order to more clearly and accurately describe the event-triggered scheme, we indicate that the current state of the system (1) is $\boldsymbol{x}^{h}(i, j), \boldsymbol{x}^{v}(i, j)$, whereas the latest transmitted state is denoted by $\boldsymbol{x}^{h}\left(i_{k}, j\right), \boldsymbol{x}^{v}\left(i, j_{l}\right)(k, l=$ $0,1,2, \ldots)$ where $i_{k}, j_{l}$ represent the event-triggered time instants and $\boldsymbol{x}^{h}\left(i_{0}, j\right)=0, \boldsymbol{x}^{v}\left(i, j_{0}\right)=0$. Then, the current state $\boldsymbol{x}^{h}(i, j), \boldsymbol{x}^{v}(i, j)$ are transmitted only when the current state of the system and the latest transmitted state satisfy the following relationship:

$$
\begin{aligned}
& {\left[\begin{array}{l}
\boldsymbol{e}^{h}(i, j) \\
\boldsymbol{e}^{v}(i, j)
\end{array}\right]^{\mathrm{T}} \boldsymbol{\Omega}\left[\begin{array}{l}
\boldsymbol{e}^{h}(i, j) \\
\boldsymbol{e}^{v}(i, j)
\end{array}\right]>} \\
& {\left[\begin{array}{l}
\boldsymbol{x}^{h}(i, j) \\
\boldsymbol{x}^{v}(i, j)
\end{array}\right]^{\mathrm{T}} \gamma \boldsymbol{\Omega}\left[\begin{array}{l}
\boldsymbol{x}^{h}(i, j) \\
\boldsymbol{x}^{v}(i, j)
\end{array}\right]}
\end{aligned}
$$

where

$$
\left[\begin{array}{l}
\boldsymbol{e}^{h}(i, j) \\
\boldsymbol{e}^{v}(i, j)
\end{array}\right]=\left[\begin{array}{l}
\boldsymbol{x}^{h}\left(i_{k}, j\right) \\
\boldsymbol{x}^{v}\left(i, j_{l}\right)
\end{array}\right]-\left[\begin{array}{l}
\boldsymbol{x}^{h}(i, j) \\
\boldsymbol{x}^{v}(i, j)
\end{array}\right]
$$

$\boldsymbol{\Omega}=\left[\begin{array}{ll}\boldsymbol{\Omega}_{h} & \\ & \boldsymbol{\Omega}_{v}\end{array}\right]$ is a positive definite weighting matrix, and $\gamma>0$ is constant scalar.

According to the event-triggered condition (6), it is obvious that before the next event-triggered time instant $i \in\left[i_{k}, i_{k+1}\right)$ and $j_{l} \in\left[j_{k}, j_{k+1}\right)$, the relationship between the current state and the latest transmitted state satisfies the following inequality:

$$
\begin{aligned}
& {\left[\begin{array}{l}
\boldsymbol{e}^{h}(i, j) \\
\boldsymbol{e}^{v}(i, j)
\end{array}\right]^{\mathrm{T}} \boldsymbol{\Omega}\left[\begin{array}{l}
\boldsymbol{e}^{h}(i, j) \\
\boldsymbol{e}^{v}(i, j)
\end{array}\right] \leqslant} \\
& {\left[\begin{array}{l}
\boldsymbol{x}^{h}(i, j) \\
\boldsymbol{x}^{v}(i, j)
\end{array}\right]^{\mathrm{T}} \gamma \boldsymbol{\Omega}\left[\begin{array}{l}
\boldsymbol{x}^{h}(i, j) \\
\boldsymbol{x}^{v}(i, j)
\end{array}\right] .}
\end{aligned}
$$

The latest transmitted state of system remains unchanged, and the state feedback control input is not updated. At this time instant, the state feedback control input is kept unchanged by the $\mathrm{ZOH}$.

For system (1), combined with the event-triggered scheme, we design the event-triggered state-feedback control law as follows:

$$
\boldsymbol{u}(i, j)=\boldsymbol{G}\left[\begin{array}{c}
\boldsymbol{x}^{h}\left(i_{k}, j\right) \\
\boldsymbol{x}^{v}\left(i, j_{l}\right)
\end{array}\right] \text {. }
$$

For system (1), define the following quadratic cost indicators:

$$
\begin{gathered}
J=\sum_{i=0}^{\infty} \sum_{j=0}^{\infty}\left\{\left[\begin{array}{l}
\boldsymbol{x}^{h}(i, j) \\
\boldsymbol{x}^{v}(i, j)
\end{array}\right]^{\mathrm{T}} \boldsymbol{Q}\left[\begin{array}{l}
\boldsymbol{x}^{h}(i, j) \\
\boldsymbol{x}^{v}(i, j)
\end{array}\right]+\right. \\
\left.\boldsymbol{u}^{\mathrm{T}}(i, j) \boldsymbol{R} \boldsymbol{u}(i, j)\right\}
\end{gathered}
$$

where $\boldsymbol{Q}$ and $\boldsymbol{R}$ are positive definite symmetric weighting matrices with appropriate dimensions.

Before proceeding further, we first introduce the following definition.

Definition 2 For uncertain 2-D system (1), considering event-triggered scheme (6), if there exists control low $\boldsymbol{u}^{*}(i, j)=\boldsymbol{G}^{*}\left[\begin{array}{c}\boldsymbol{x}^{h}\left(i_{k}, j\right) \\ \boldsymbol{x}^{v}\left(i, j_{l}\right)\end{array}\right]$ and a positive scalar $J^{*}$ such that for all uncertainties of the system, the event-triggered closed-loop system is robust asymptotically stable and the cost function (9) satisfies $J \leqslant J^{*}$, then $J^{*}$ is said to be a cost upper bound of the system and $\boldsymbol{u}^{*}(i, j)$ is said to be an event-triggered guaranteed cost control low for the uncertain 2-D system (1).

Then, the main research problem in this paper is that for the uncertain 2-D nonlinear discrete system (1) based on the Roesser model, considering the event-triggered condition (6), design a guaranteed cost state-feedback control law $\boldsymbol{u}^{*}(i, j)=\boldsymbol{G}^{*}\left[\begin{array}{c}\boldsymbol{x}^{h}\left(i_{k}, j\right) \\ \boldsymbol{x}^{v}\left(i, j_{l}\right)\end{array}\right]$ to make the uncertain closed-loop system robustly asymptotically stable, while there is a positive scalar $J^{*}$ satisfying $J \leqslant J^{*}$.

\section{Main results}

Give the lemma needed in the proof of the subsequent theorem: 
Lemma 1 [25] Given the matrices $\boldsymbol{Y}, \boldsymbol{H}$ and $\boldsymbol{E}$ of the appropriate dimension, where $\boldsymbol{Y}$ is symmetric, then

$$
\boldsymbol{Y}+\boldsymbol{H} \boldsymbol{F} \boldsymbol{E}+\boldsymbol{E}^{\mathrm{T}} \boldsymbol{F}^{\mathrm{T}} \boldsymbol{H}^{\mathrm{T}}<\mathbf{0}
$$

holds for all matrices $\boldsymbol{F}$ satisfying $\boldsymbol{F}^{\mathrm{T}} \boldsymbol{F} \leqslant \boldsymbol{I}$ if and only if there is a constant $\varepsilon>0$ such that

$$
\boldsymbol{Y}+\varepsilon \boldsymbol{H} \boldsymbol{H}^{\mathrm{T}}+\varepsilon^{-1} \boldsymbol{E}^{\mathrm{T}} \boldsymbol{E}<\mathbf{0} .
$$

First, considering the event-triggered condition (6) in Section 2, Theorem 1 gives the sufficient conditions for the robust asymptotic stability of uncertain 2-D nonlinear systems (1).

Theorem 1 For the 2-D uncertain nonlinear system (1), event-triggered parameters $\gamma$ and feedback control gain matrices $\boldsymbol{G}$ are known. If there exist symmetric matrices $\boldsymbol{P}=\operatorname{diag}\left\{\boldsymbol{P}_{h}, \boldsymbol{P}_{v}\right\}>\mathbf{0}, \boldsymbol{\Omega}=\operatorname{diag}\left\{\boldsymbol{\Omega}_{h}, \boldsymbol{\Omega}_{v}\right\}>\mathbf{0}$ with appropriate dimensions and a scalar $\mu>0$ satisfying the following inequality:

$$
\boldsymbol{\Theta}=\left[\begin{array}{ccc}
\boldsymbol{\Theta}_{11} & \boldsymbol{\Theta}_{12} & \boldsymbol{\Theta}_{13} \\
* & \boldsymbol{\Theta}_{22} & \boldsymbol{\Theta}_{23} \\
* & * & \boldsymbol{\Theta}_{33}
\end{array}\right]<\mathbf{0}
$$

where

$$
\begin{gathered}
\boldsymbol{\Theta}_{11}=\boldsymbol{\Pi}_{1}^{\mathrm{T}} \boldsymbol{P} \boldsymbol{\Pi}_{1}-\boldsymbol{P}+\gamma \boldsymbol{\Omega} \\
\boldsymbol{\Theta}_{12}=\boldsymbol{\Pi}_{1}^{\mathrm{T}} \boldsymbol{P}\left(\boldsymbol{B}_{1} \boldsymbol{G}+\boldsymbol{D} \boldsymbol{F} \boldsymbol{E}_{2} \boldsymbol{G}\right) \\
\boldsymbol{\Theta}_{13}=\boldsymbol{\Pi}_{1}^{\mathrm{T}} \boldsymbol{P}\left(\boldsymbol{B}+\boldsymbol{D} \boldsymbol{F} \boldsymbol{E}_{3}\right)+\mu \boldsymbol{C}^{\mathrm{T}} \boldsymbol{K} \\
\boldsymbol{\Theta}_{22}=\left(\boldsymbol{B}_{1} \boldsymbol{G}+\boldsymbol{D} \boldsymbol{F} \boldsymbol{E}_{2} \boldsymbol{G}\right)^{\mathrm{T}} \boldsymbol{P}\left(\boldsymbol{B}_{1} \boldsymbol{G}+\right. \\
\left.\boldsymbol{D} \boldsymbol{F} \boldsymbol{E}_{2} \boldsymbol{G}\right)-\boldsymbol{\Omega} \\
\boldsymbol{\Theta}_{23}=\left(\boldsymbol{B}_{1} \boldsymbol{G}+\boldsymbol{D} \boldsymbol{F} \boldsymbol{E}_{2} \boldsymbol{G}\right)^{\mathrm{T}} \boldsymbol{P}\left(\boldsymbol{B}+\boldsymbol{D} \boldsymbol{F} \boldsymbol{E}_{3}\right) \\
\boldsymbol{\Theta}_{33}=\left(\boldsymbol{B}+\boldsymbol{D} \boldsymbol{F} \boldsymbol{E}_{3}\right)^{\mathrm{T}} \boldsymbol{P}\left(\boldsymbol{B}+\boldsymbol{D} \boldsymbol{F} \boldsymbol{E}_{3}\right)-2 \mu \boldsymbol{I} \\
\boldsymbol{\Pi}_{1}=\boldsymbol{A}+\boldsymbol{B}_{1} \boldsymbol{G}+\boldsymbol{B} \boldsymbol{K}_{1} \boldsymbol{C}+\boldsymbol{D} \boldsymbol{F} \boldsymbol{E}_{1}+ \\
\boldsymbol{D} \boldsymbol{F} \boldsymbol{E}_{2} \boldsymbol{G}+\boldsymbol{D} \boldsymbol{F} \boldsymbol{E}_{3} \boldsymbol{K}_{1} \boldsymbol{C} .
\end{gathered}
$$

Then the closed-loop system is robust asymptotically stable for all $\psi(\boldsymbol{v}) \in\left[\boldsymbol{K}_{1}, \boldsymbol{K}_{2}\right]$.

Proof According to Definition 1 in Section 2, we can rewrite $\psi(\boldsymbol{v})$ as follows:

$$
\psi(\boldsymbol{v})=\psi_{n}(\boldsymbol{v})+\boldsymbol{K}_{1} \boldsymbol{v}
$$

where $\psi_{n}(\boldsymbol{v}) \in \phi$ represents a nonlinear part given by

$$
\phi=\left\{\left(\psi_{n}(\boldsymbol{v})\right)^{\mathrm{T}}\left(\psi_{n}(\boldsymbol{v})-\boldsymbol{K} \boldsymbol{v}\right) \leqslant 0\right\} .
$$

Since $\psi$ belongs to the sector $\left[\boldsymbol{K}_{1}, \boldsymbol{K}_{2}\right]$, then according to (2), the system (1) becomes

$$
\begin{gathered}
{\left[\begin{array}{l}
\boldsymbol{x}^{h}(i+1, j) \\
\boldsymbol{x}^{v}(i, j+1)
\end{array}\right]=\boldsymbol{\Pi}_{1}\left[\begin{array}{l}
\boldsymbol{x}^{h}(i, j) \\
\boldsymbol{x}^{v}(i, j)
\end{array}\right]+} \\
\left(\boldsymbol{B}_{1}+\boldsymbol{D} \boldsymbol{F} \boldsymbol{E}_{2}\right) \boldsymbol{G}\left[\begin{array}{l}
\boldsymbol{e}^{h}(i, j) \\
\boldsymbol{e}^{v}(i, j)
\end{array}\right]+ \\
\left(\boldsymbol{B}+\boldsymbol{D} \boldsymbol{F} \boldsymbol{E}_{3}\right) \psi_{n}(\boldsymbol{v}) .
\end{gathered}
$$

Select the Lyapunov function as

$$
V(i, j)=\left[\begin{array}{l}
\boldsymbol{x}^{h}(i, j) \\
\boldsymbol{x}^{v}(i, j)
\end{array}\right]^{\mathrm{T}} \boldsymbol{P}\left[\begin{array}{l}
\boldsymbol{x}^{h}(i, j) \\
\boldsymbol{x}^{v}(i, j)
\end{array}\right] .
$$

Then

$$
\begin{gathered}
\Delta V(i, j)=V(i+1, j+1)-V(i, j)= \\
{\left[\begin{array}{l}
\boldsymbol{x}^{h}(i+1, j) \\
\boldsymbol{x}^{v}(i, j+1)
\end{array}\right]^{\mathrm{T}} \boldsymbol{P}\left[\begin{array}{l}
\boldsymbol{x}^{h}(i+1, j) \\
\boldsymbol{x}^{v}(i, j+1)
\end{array}\right]-} \\
{\left[\begin{array}{l}
\boldsymbol{x}^{h}(i, j) \\
\boldsymbol{x}^{v}(i, j)
\end{array}\right]^{\mathrm{T}} \boldsymbol{P}\left[\begin{array}{l}
\boldsymbol{x}^{h}(i, j) \\
\boldsymbol{x}^{v}(i, j)
\end{array}\right] .}
\end{gathered}
$$

According to (7) and (11), we can obtain that for any $\mu>0$,

$$
\begin{gathered}
\Delta V(i, j)<\left\{\boldsymbol{\Pi}_{1} \boldsymbol{x}(i, j)+\left(\boldsymbol{B}+\boldsymbol{D} \boldsymbol{F} \boldsymbol{E}_{3}\right)\right. \\
\left.\psi_{n}(\boldsymbol{v})+\left(\boldsymbol{B}_{1} \boldsymbol{G}+\boldsymbol{D} \boldsymbol{F} \boldsymbol{E}_{2} \boldsymbol{G}\right) \boldsymbol{e}(i, j)\right\}^{\mathrm{T}} . \\
\boldsymbol{P}\left\{\boldsymbol{\Pi}_{1} \boldsymbol{x}(i, j)+\left(\boldsymbol{B}+\boldsymbol{D} \boldsymbol{F} \boldsymbol{E}_{3}\right) \psi_{n}(\boldsymbol{v})+\right. \\
\left.\left(\boldsymbol{B}_{1} \boldsymbol{G}+\boldsymbol{D} \boldsymbol{F} \boldsymbol{E}_{2} \boldsymbol{G}\right) \boldsymbol{e}(i, j)\right\}-\boldsymbol{x}(i, j)^{\mathrm{T}} . \\
\boldsymbol{P} \boldsymbol{x}(i, j)+\boldsymbol{x}(i, j)^{\mathrm{T}} \gamma \boldsymbol{\Omega} \boldsymbol{x}(i, j)- \\
\boldsymbol{e}(i, j)^{\mathrm{T}} \boldsymbol{\Omega} \boldsymbol{e}(i, j)-2 \mu \psi_{n}(\boldsymbol{v})^{\mathrm{T}} . \\
\left(\psi_{n}(\boldsymbol{v})-\boldsymbol{K} \boldsymbol{v}\right)=\left[\begin{array}{l}
\boldsymbol{x}(i, j) \\
\boldsymbol{e}(i, j) \\
\psi_{n}(\boldsymbol{v})
\end{array}\right]^{\mathrm{T}} \boldsymbol{\Theta}\left[\begin{array}{l}
\boldsymbol{x}(i, j) \\
\boldsymbol{e}(i, j) \\
\psi_{n}(\boldsymbol{v})
\end{array}\right]
\end{gathered}
$$

where

$$
\begin{aligned}
& \boldsymbol{x}(i, j)=\left[\begin{array}{l}
\boldsymbol{x}^{h}(i, j) \\
\boldsymbol{x}^{v}(i, j)
\end{array}\right] \\
& \boldsymbol{e}(i, j)=\left[\begin{array}{l}
\boldsymbol{e}^{h}(i, j) \\
\boldsymbol{e}^{v}(i, j)
\end{array}\right] .
\end{aligned}
$$

By (10), $\Delta V(i, j)<\mathbf{0}$. It is known that the uncertain 2-D nonlinear system with the event-triggered scheme is robustly asymptotically stable for all $\psi(\boldsymbol{v}) \in\left[\boldsymbol{K}_{1}, \boldsymbol{K}_{2}\right]$.

Remark 1 Theorem 1 gives a sufficient condition for the robust asymptotic stability of an event-triggered 2-D nonlinear system under the assumption that the control gain matrix $G$ is known. $\gamma>0$ is the event-triggered parameter. When $\gamma=0$, the event-triggered scheme (6) degrades to the common period-triggering scheme. The condition in Theorem 1 becomes a sufficient condition for the robust asymptotic stability of the period-triggering scheme 2-D nonlinear system.

Then, based on Lemma 1 and Theorem 1, using LMI techniques, we can derive the following LMI in Theorem 2, which can be used to design an event-triggered guaranteed cost controller for 2-D uncertain nonlinear system (1).

Theorem 2 For the system (1) with event-triggered condition (6), if there exist symmetric matrices $\boldsymbol{X}=$ $\operatorname{diag}\left\{\boldsymbol{X}_{h}, \boldsymbol{X}_{v}\right\}, \overline{\boldsymbol{\Omega}}, \boldsymbol{Z}, \boldsymbol{W}$ with appropriate dimensions and scalars $\varepsilon>0, \alpha>0$ satisfing the following LMI: 


$$
\left[\begin{array}{cccccc}
\gamma \overline{\boldsymbol{\Omega}}-\boldsymbol{X}+\boldsymbol{W} & 0 & \boldsymbol{X} \boldsymbol{C}^{\mathrm{T}} \boldsymbol{K} & \boldsymbol{X}\left(\boldsymbol{A}+\boldsymbol{B} \boldsymbol{K}_{1} \boldsymbol{C}\right)^{\mathrm{T}}+\boldsymbol{Z}^{\mathrm{T}} \boldsymbol{B}_{1}^{\mathrm{T}} & \boldsymbol{Z}^{\mathrm{T}} & \boldsymbol{X} \boldsymbol{E}_{1}^{\mathrm{T}}+\boldsymbol{Z}^{\mathrm{T}} \boldsymbol{E}_{2}^{\mathrm{T}}+\boldsymbol{X}\left(\boldsymbol{E}_{3} \boldsymbol{K}_{1} \boldsymbol{C}\right)^{\mathrm{T}} \\
* & -\overline{\boldsymbol{\Omega}} & 0 & \boldsymbol{Z}^{\mathrm{T}} \boldsymbol{B}_{1}^{\mathrm{T}} & \boldsymbol{Z}^{\mathrm{T}} & \boldsymbol{Z}^{\mathrm{T}} \boldsymbol{E}_{2}^{\mathrm{T}} \\
* & * & -2 \alpha \boldsymbol{I} & \alpha \boldsymbol{B}^{\mathrm{T}} & 0 & \boldsymbol{X}_{3}^{\mathrm{T}} \\
* & * & * & -\boldsymbol{X}+\varepsilon \boldsymbol{D} \boldsymbol{D}^{\mathrm{T}} & 0 & 0 \\
* & * & * & * & -\boldsymbol{V} & 0 \\
* & * & * & * & * & -\varepsilon \boldsymbol{I}
\end{array}\right]
$$

Then there exists a state-feedback control low $\boldsymbol{u}^{*}(i, j)=\boldsymbol{G}^{*}\left[\begin{array}{c}\boldsymbol{x}^{h}\left(i_{k}, j\right) \\ \boldsymbol{x}^{v}\left(i, j_{l}\right)\end{array}\right]$ that solves the addressed robust guaranteed cost control problem. In this situation, a suitable control gain is designed by $\boldsymbol{G}^{*}=\boldsymbol{Z} \boldsymbol{X}^{-1}$ and the weighting matrix of the event-triggered condition is designed by $\bar{\Omega}=\boldsymbol{X}^{-1} \tilde{\boldsymbol{\Omega}} \boldsymbol{X}^{-1}$. Moreover, the closed-loop cost function satisfies

$$
J \leqslant J^{*}=n \lambda_{\max }\left(\boldsymbol{L}^{\mathrm{T}} \boldsymbol{X}_{h}^{-1} \boldsymbol{L}\right)+m \lambda_{\max }\left(\boldsymbol{L}^{\mathrm{T}} \boldsymbol{X}_{v}^{-1} \boldsymbol{L}\right) .
$$

Proof According to Definition 2 and Theorem 1, there exists guaranteed cost control law $\boldsymbol{G}$ with event-triggered scheme (6), if there exists symmetric matrices $Q>0$, $\boldsymbol{R}>\mathbf{0}, \boldsymbol{P}=\left[\begin{array}{ll}\boldsymbol{P}_{h} & \\ & \boldsymbol{P}_{v}\end{array}\right]>\mathbf{0}, \boldsymbol{\Omega}=\left[\begin{array}{ll}\boldsymbol{\Omega}_{h} & \\ & \boldsymbol{\Omega}_{v}\end{array}\right]>\mathbf{0}$ and a scalar $\mu>0$ satisfying

$$
\left[\begin{array}{l}
\boldsymbol{x}(i, j) \\
\boldsymbol{e}(i, j) \\
\psi_{n}(\boldsymbol{v})
\end{array}\right]^{\mathrm{T}}\left[\begin{array}{ccc}
\boldsymbol{\Pi}_{11} & \boldsymbol{\Pi}_{12} & \boldsymbol{\Theta}_{13} \\
* & \boldsymbol{\Pi}_{22} & \boldsymbol{\Theta}_{23} \\
* & * & \boldsymbol{\Theta}_{33}
\end{array}\right]\left[\begin{array}{l}
\boldsymbol{x}(i, j) \\
\boldsymbol{e}(i, j) \\
\psi_{n}(\boldsymbol{v})
\end{array}\right]<\mathbf{0}
$$

where $\boldsymbol{\Theta}_{13}, \boldsymbol{\Theta}_{23}, \boldsymbol{\Theta}_{33}$ are defined in Theorem 1,

$$
\begin{gathered}
\boldsymbol{\Pi}_{11}=\boldsymbol{\Theta}_{11}+\boldsymbol{Q}+\boldsymbol{G}^{\mathrm{T}} \boldsymbol{R} \boldsymbol{G} \\
\boldsymbol{\Pi}_{12}=\boldsymbol{\Theta}_{12}+\boldsymbol{G}^{\mathrm{T}} \boldsymbol{R} \boldsymbol{G} \\
\boldsymbol{\Pi}_{22}=\boldsymbol{\Theta}_{22}+\boldsymbol{G}^{\mathrm{T}} \boldsymbol{R} \boldsymbol{G} .
\end{gathered}
$$

First, the proof method of the upper bound of the cost indicator is given. From (13) we have

$$
\begin{gathered}
{\left[\begin{array}{l}
\boldsymbol{x}^{h}(i, j) \\
\boldsymbol{x}^{v}(i, j)
\end{array}\right]^{\mathrm{T}} \boldsymbol{Q}\left[\begin{array}{l}
\boldsymbol{x}^{h}(i, j) \\
\boldsymbol{x}^{v}(i, j)
\end{array}\right]+} \\
\boldsymbol{u}^{\mathrm{T}}(i, j) \boldsymbol{R u}(i, j)<-\Delta V(i, j)<\mathbf{0 .} .
\end{gathered}
$$

Summing both sides of the above inequality over $i, j=$ $0 \rightarrow \infty$ yields

$$
\begin{gathered}
J<-\sum_{i=0}^{\infty} \sum_{j=0}^{\infty} \Delta V(i, j)= \\
\sum_{j=0}^{\infty} \boldsymbol{x}^{h \mathrm{~T}}(0, j) \boldsymbol{P}_{h} \boldsymbol{x}^{h}(0, j)+ \\
\sum_{i=0}^{\infty} \boldsymbol{x}^{v \mathrm{~T}}(i, 0) \boldsymbol{P}_{v} \boldsymbol{x}^{v}(i, 0) .
\end{gathered}
$$

According to Assumption 1, the boundary conditions of the system, (14) can be further equivalent to the following inequalities:

$$
J \leqslant n \lambda_{\max }\left(\boldsymbol{L}^{\mathrm{T}} \boldsymbol{P}_{h} \boldsymbol{L}\right)+m \lambda_{\max }\left(\boldsymbol{L}^{\mathrm{T}} \boldsymbol{P}_{v} \boldsymbol{L}\right) .
$$

Next, the design method of the guaranteed cost state feedback control law under the event-triggered condition is given. Inequality (13) can be rewritten as follows:

$$
\boldsymbol{\zeta}^{\mathrm{T}} \boldsymbol{P} \boldsymbol{\zeta}+\left[\begin{array}{ccc}
\gamma \boldsymbol{\Omega}-\boldsymbol{P}+\boldsymbol{Q}+\boldsymbol{G}^{\mathrm{T}} \boldsymbol{R} \boldsymbol{G} & \boldsymbol{G}^{\mathrm{T}} \boldsymbol{R} \boldsymbol{G} & \mu \boldsymbol{C}^{\mathrm{T}} \boldsymbol{K} \\
* & -\boldsymbol{\Omega}+\boldsymbol{G}^{\mathrm{T}} \boldsymbol{R} \boldsymbol{G} & 0 \\
* & * & -2 \mu \boldsymbol{I}
\end{array}\right]<\mathbf{0}
$$

where

$$
\boldsymbol{\zeta}=\left[\begin{array}{lll}
\boldsymbol{\Pi}_{1} & \boldsymbol{B}_{1} \boldsymbol{G}+\boldsymbol{D F} \boldsymbol{E}_{2} \boldsymbol{G} & \boldsymbol{B}+\boldsymbol{D F} \boldsymbol{E}_{3}
\end{array}\right]
$$

Using Schur complement, (15) can be rewritten as

$$
\left[\begin{array}{cccc}
\gamma \boldsymbol{\Omega}-\boldsymbol{P}+\boldsymbol{Q}+\boldsymbol{G}^{\mathrm{T}} \boldsymbol{R} \boldsymbol{G} & \boldsymbol{G}^{\mathrm{T}} \boldsymbol{R} \boldsymbol{G} & \mu \boldsymbol{C}^{\mathrm{T}} \boldsymbol{K} & \boldsymbol{\Pi}_{1}^{\mathrm{T}} \\
* & -\boldsymbol{\Omega}+\boldsymbol{G}^{\mathrm{T}} \boldsymbol{R} \boldsymbol{G} & 0 & \left(\boldsymbol{B}_{1} \boldsymbol{G}+\boldsymbol{D} \boldsymbol{F} \boldsymbol{E}_{2} \boldsymbol{G}\right)^{\mathrm{T}} \\
* & * & -2 \mu \boldsymbol{I} & \left(\boldsymbol{B}+\boldsymbol{D} \boldsymbol{F} \boldsymbol{E}_{3}\right)^{\mathrm{T}} \\
* & * & * & -\boldsymbol{P}^{-1}
\end{array}\right]<\mathbf{0} .
$$

Then, (16) can be further rewritten as follows:

$$
\boldsymbol{Y}+\boldsymbol{H} \boldsymbol{F} \boldsymbol{E}+\boldsymbol{E}^{\mathrm{T}} \boldsymbol{F}^{\mathrm{T}} \boldsymbol{H}^{\mathrm{T}}<\mathbf{0}
$$


where

$$
\boldsymbol{Y}=\left[\begin{array}{cccc}
\gamma \boldsymbol{\Omega}-\boldsymbol{P}+\boldsymbol{Q}+\boldsymbol{G}^{\mathrm{T}} \boldsymbol{R} \boldsymbol{G} & \boldsymbol{G}^{\mathrm{T}} \boldsymbol{R} \boldsymbol{G} & \mu \boldsymbol{C}^{\mathrm{T}} \boldsymbol{K} & \left(\boldsymbol{A}+\boldsymbol{B} \boldsymbol{K}_{1} \boldsymbol{C}+\boldsymbol{B}_{1} \boldsymbol{G}\right)^{\mathrm{T}} \\
& -\boldsymbol{\Omega}+\boldsymbol{G}^{\mathrm{T}} \boldsymbol{R} \boldsymbol{G} & 0 & \left(\boldsymbol{B}_{1} \boldsymbol{G}\right)^{\mathrm{T}} \\
* & * & -2 \mu \boldsymbol{I} & \boldsymbol{B}^{\mathrm{T}} \\
* & * & * & -\boldsymbol{P}^{-1}
\end{array}\right]
$$

equivalent to

$$
\begin{gathered}
\boldsymbol{H}=\left[0,0,0, \boldsymbol{D}^{\mathrm{T}}\right]^{\mathrm{T}} \\
\boldsymbol{E}=\left[\begin{array}{llll}
\boldsymbol{E}_{1}+\boldsymbol{E}_{2} \boldsymbol{G}+\boldsymbol{E}_{3} \boldsymbol{K}_{1} \boldsymbol{C} & \boldsymbol{E}_{2} \boldsymbol{G} & \boldsymbol{E}_{3} & 0
\end{array}\right] .
\end{gathered}
$$

According to Lemma 1, for all matrices $\boldsymbol{F}$ satisfying $\boldsymbol{F}^{\mathrm{T}} \boldsymbol{F} \leqslant \boldsymbol{I}$, there is a constant $\varepsilon>0$, which makes (17)

$$
\boldsymbol{Y}+\varepsilon \boldsymbol{H} \boldsymbol{H}^{\mathrm{T}}+\varepsilon^{-1} \boldsymbol{E}^{\mathrm{T}} \boldsymbol{E}<\mathbf{0} .
$$

Using Schur complement, (18) can be rewritten as

$$
\left[\begin{array}{cccccc}
\gamma \boldsymbol{\Omega}-\boldsymbol{P}+\boldsymbol{Q} & 0 & \mu \boldsymbol{C}^{\mathrm{T}} \boldsymbol{K} & \left(\boldsymbol{A}+\boldsymbol{B} \boldsymbol{K}_{1} \boldsymbol{C}\right)^{\mathrm{T}}+\boldsymbol{G}^{\mathrm{T}} \boldsymbol{B}_{1}^{\mathrm{T}} & \boldsymbol{G}^{\mathrm{T}} & \left(\boldsymbol{E}_{1}+\boldsymbol{E}_{2} \boldsymbol{G}+\boldsymbol{E}_{3} \boldsymbol{K}_{1} \boldsymbol{C}\right)^{\mathrm{T}} \\
* & -\boldsymbol{\Omega} & 0 & \boldsymbol{G}^{\mathrm{T}} \boldsymbol{B}_{1}^{\mathrm{T}} & \boldsymbol{G}^{\mathrm{T}} & \left(\boldsymbol{E}_{2} \boldsymbol{G}\right)^{\mathrm{T}} \\
* & * & -2 \mu \boldsymbol{I} & \boldsymbol{B}^{\mathrm{T}} & 0 & \boldsymbol{E}_{3} \\
* & * & * & -\boldsymbol{P}^{-1}+\varepsilon \mathbf{D D}^{\mathrm{T}} & 0 & 0 \\
* & * & * & * & -\boldsymbol{R}^{-1} & 0 \\
* & * & * & * & * & -\varepsilon \boldsymbol{I}
\end{array}\right]<\mathbf{0}
$$

Define $\boldsymbol{X}=\boldsymbol{P}^{-1}, \overline{\boldsymbol{\Omega}}=\boldsymbol{X}^{\mathrm{T}} \boldsymbol{\Omega} \boldsymbol{X}, \boldsymbol{Z}=\boldsymbol{G} \boldsymbol{X}$, premultiplying and postmultiplying (19) by the matrix $\operatorname{diag}\left\{\boldsymbol{P}^{-1}, \boldsymbol{P}^{-1}, \mu^{-1}, \boldsymbol{I}, \boldsymbol{I}, \boldsymbol{I}\right\}$, the condition (12) in Theorem 2 can be obtained.

\section{Illustrative examples}

This section demonstrates the effectiveness of the proposed control algorithm through a numerical simulation example.

Consider system (1) with the following system matrices:

$$
\begin{gathered}
\boldsymbol{A}=\left[\begin{array}{cc}
0.8 & 0.21 \\
0.01 & 0.9
\end{array}\right], \boldsymbol{B}=\left[\begin{array}{cc}
0.1 & 0 \\
0.01 & 0.02
\end{array}\right] \\
\boldsymbol{B}_{1}=\left[\begin{array}{cc}
0.03 & 0 \\
0.1 & 0.1
\end{array}\right], \boldsymbol{C}=\left[\begin{array}{cc}
0.2 & 0.08 \\
0.17 & 0.22
\end{array}\right] \\
\boldsymbol{K}_{1}=\left[\begin{array}{cc}
0.1 & 0 \\
0 & 0.1
\end{array}\right], \boldsymbol{K}_{2}=\left[\begin{array}{cc}
0.2 & 0 \\
0 & 0.2
\end{array}\right] \\
\boldsymbol{D}=\left[\begin{array}{cc}
0.1 & 0.1 \\
0 & 0.1
\end{array}\right], \boldsymbol{E}_{1}=\left[\begin{array}{cc}
0.1 & 0.1 \\
0.1 & 0.1
\end{array}\right] \\
\boldsymbol{E}_{2}=\left[\begin{array}{cc}
0.1 & 0 \\
0.1 & 0.1
\end{array}\right], \boldsymbol{E}_{3}=\left[\begin{array}{cc}
0.1 & 0.1 \\
0 & 0.1
\end{array}\right]
\end{gathered}
$$

and the initial conditions satisfy (4) and (5) with $m=$ $n=2, \boldsymbol{L}=0.1$, the cost function is given by (9) with $\boldsymbol{Q}=\boldsymbol{R}=\operatorname{diag}\{1,1\}$.

According to the system parameters given above, the event-triggered state-feedback guaranteed cost controller of the system (1) can be designed by using Theorem 2 in Section 3, then the closed-loop system is robustly asymptotically stable. In order to further demonstrate the effectiveness and superiority of the proposed algorithm, we consider the following two cases, one using the robust guaranteed cost control method in [12], and the other using the event-triggered robust guaranteed cost control method proposed in this paper. The system parameters of Case 2 and the initial conditions of the system are the same as those of Case 1 .

Case 1 According to the linear inequality condition of Theorem 2 in [12], the LMI tool in MATLAB is used to solve the feasible solution, and the corresponding parameters are obtained as follows:

$$
\boldsymbol{G}^{*}=\left[\begin{array}{ll}
-0.105 & -0.219 \\
-0.169 & -0.422
\end{array}\right], \quad J^{*}=8.898 .
$$

Then, under the control of the designed controller, the state response of the system is as shown in Fig. 2 and Fig. 3. The control inputs are shown in Fig. 4 and Fig. 5.

Case 2 Under event-triggered scheme (6) $(\gamma=0.8)$. According to the linear inequality condition (12) in Theorem 2, the LMI tool in MATLAB is used to solve the feasible solution, and the corresponding parameters are obtained as follows:

$$
\begin{gathered}
\boldsymbol{G}^{*}=\left[\begin{array}{ll}
-0.096 & -0.189 \\
-0.105 & -0.448
\end{array}\right] \\
\overline{\boldsymbol{\Omega}}=\left[\begin{array}{ll}
0.385 & \\
& 1.236
\end{array}\right] \\
J^{*}=14.840 .
\end{gathered}
$$

Then, under the control of the designed controller, the state response of the system is as shown in Fig. 6 and Fig. 7. The control inputs are shown in Fig. 8 and Fig. 9. 


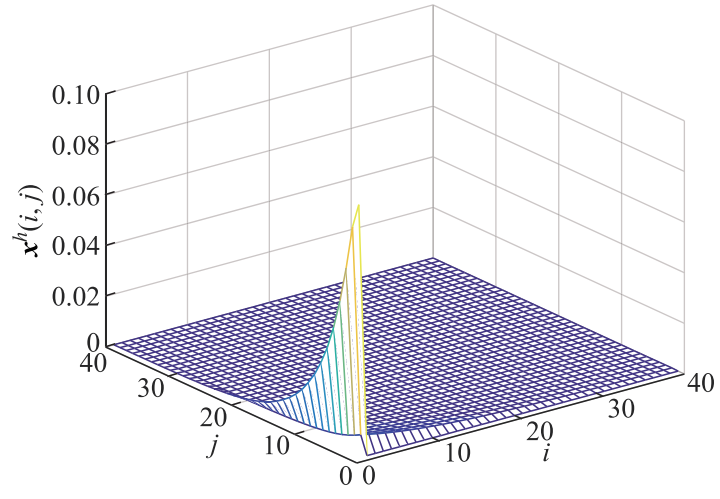

Fig. 2 State response of $x^{h}(i, j)$ in Case 1

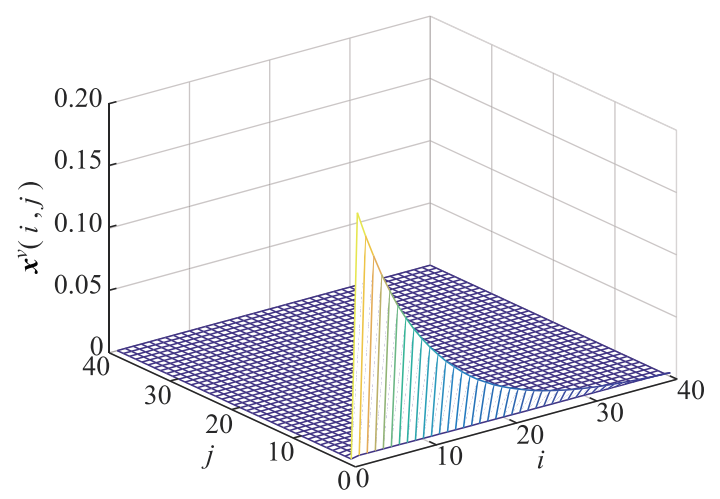

Fig. 3 State response of $x^{v}(i, j)$ in Case 1

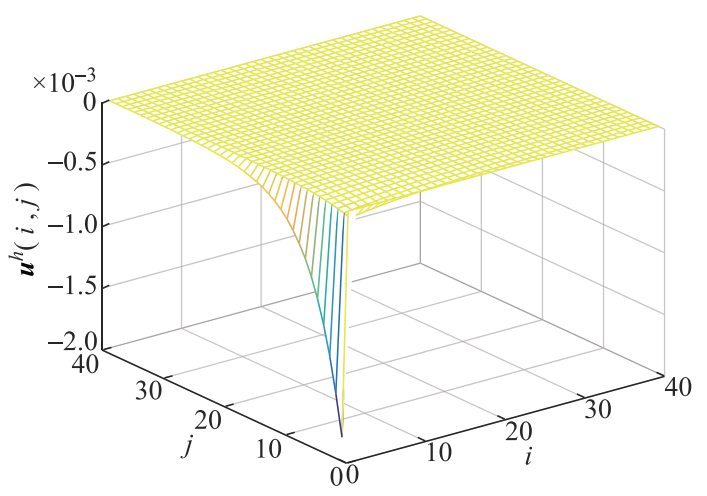

Fig. 4 Control input of $u^{h}$ in Case 1

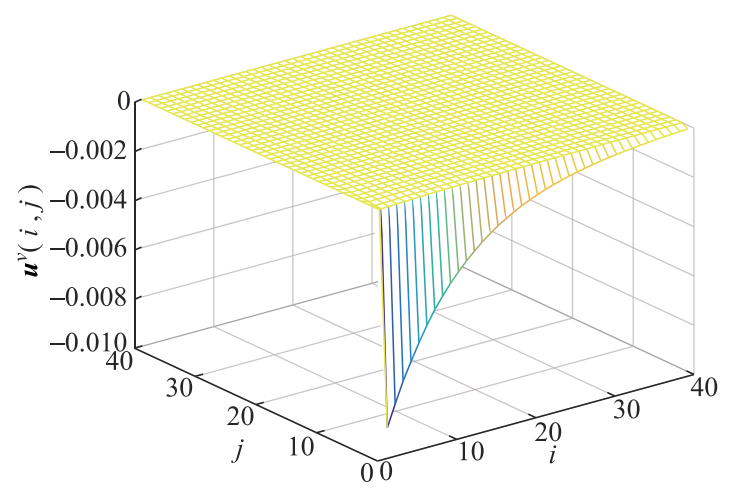

Fig. 5 Control input of $u^{v}$ in Case 1

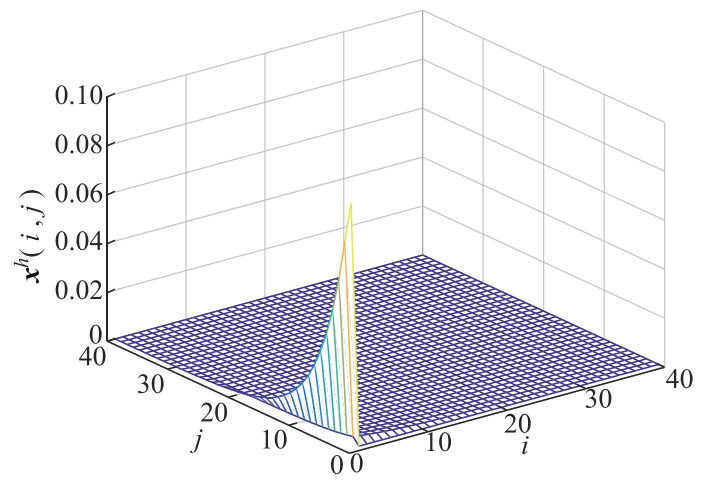

Fig. 6 State response of $x^{h}(i, j)$ in Case 2

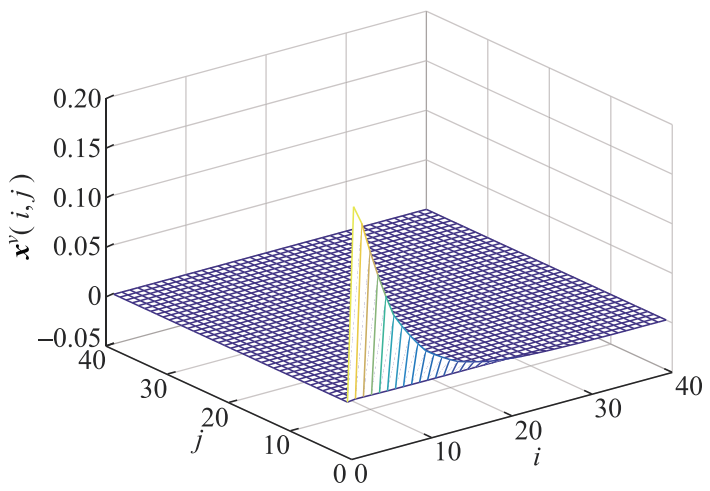

Fig. 7 State response of $x^{v}(i, j)$ in Case 2

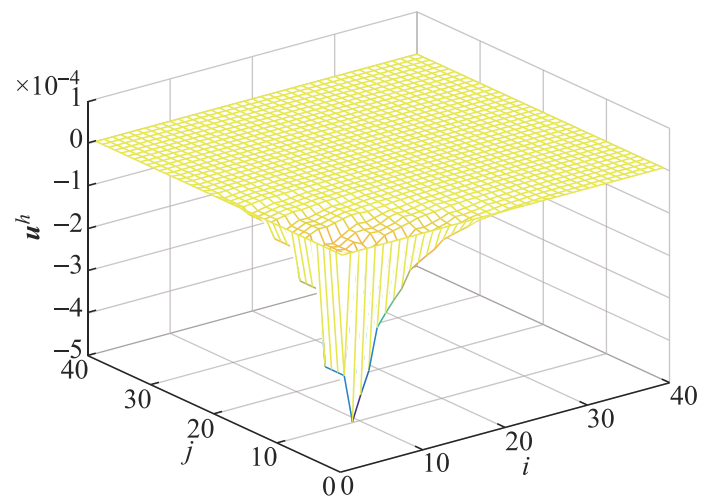

Fig. 8 Control input of $u^{h}$ in Case 2

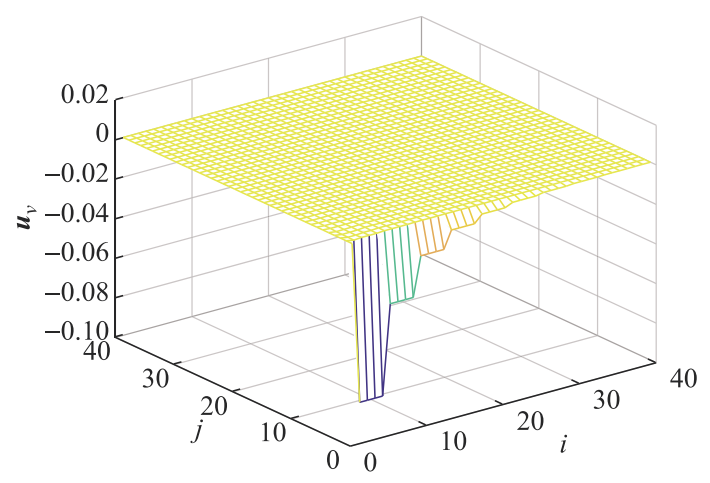

Fig. 9 Control input of $u^{v}$ in Case 2 
By analyzing the simulation results of Case 1 and Case 2, it can be concluded that the control method proposed in this paper has certain advantages.

Firstly, comparing the state response simulation results of Case 1 and Case 2, the control method proposed in this paper has faster response speed and can stabilize the system faster than the algorithm in [12]. In addition, the control method proposed in this paper can also get lower cost function indicators to achieve better performance.

Secondly, in Case 2, only 390 sampling moments need to transmit the data of $\boldsymbol{x}^{h}(i, j)$ in the sensor to the controller. Similarly, only 415 sampling moments are needed to transmit the data of $\boldsymbol{x}^{v}(i, j)$ in the sensor to the controller. The data transmission rate of $\boldsymbol{x}^{h}(i, j)$ is $24.38 \%$, and the data transmission rate of $\boldsymbol{x}^{v}(i, j)$ is $25.94 \%$. It can be seen clearly that the state data of each sampling moment is transmitted to the controller and the control input is updated at each sampling instant in Case 1, however, some unnecessary data transmission is reduced in Case 2, the control method of this paper can effectively save communication resources and computing resources.

In summary, the control method of this paper can not only improve the performance of the system but also save communication resources and reduce data transmission.

In order to further show the relationship among eventtriggered parameters, data transmission amount and data transmission rate, let $\gamma$ be $0,0.2,0.4,0.6$, and 0.8 respectively, and the data corresponding to the values of different trigger parameters are listed in Table 1. Among them, Nh represents the number of $\boldsymbol{x}^{h}(i, j)$ data transmission, $\mathrm{Nv}$ represents the number of $\boldsymbol{x}^{v}(i, j)$ data transmission, $\mathrm{Rh}$ represents the data transmission rate of $\boldsymbol{x}^{h}(i, j)$, and $\mathrm{Rv}$ represents the data transmission rate of $\boldsymbol{x}^{v}(i, j)$. It can be seen that as the triggering parameter is gradually increased, the data transmission amount is gradually reduced, and the data transmission rate is lowered.

Table 1 Some computation results for different values of $\gamma$

\begin{tabular}{cccccc}
\hline$\gamma$ & 0 & 0.2 & 0.4 & 0.6 & 0.8 \\
\hline $\mathrm{Nh}$ & 1600 & 800 & 531 & 469 & 390 \\
$\mathrm{Nv}$ & 1600 & 653 & 520 & 400 & 324 \\
$\mathrm{Rh}$ & $100 \%$ & $50 \%$ & $33.19 \%$ & $29.31 \%$ & $24.38 \%$ \\
$\mathrm{Rv}$ & $100 \%$ & $40.81 \%$ & $32.5 \%$ & $25 \%$ & $20.25 \%$ \\
\hline
\end{tabular}

\section{Conclusions}

In this paper, we investigate the problem of guaranteed cost control for a class of 2-D nonlinear systems with the event-triggered scheme, uncertain parameters and sector nonlinearities. The event-triggered conditions based on 2D nonlinear discrete systems are defined, and then the sufficient conditions for robust stability of 2-D nonlinear dis- crete systems under the event-triggered scheme are given. By using the LMI technique, these conditions are transformed into LMI, which can give the design method of the event-triggered robust guaranteed cost controller. Numerical simulation results verify the effectiveness of the proposed approach. The controller design method presented in this paper has certain versatility. Under certain circumstances, it can also be used to solve other related network control problems.

\section{References}

[1] LU M C, HSU C C, YU Y L. Image-based system for measuring objects on an oblique plane and its applications in 2-D localization. IEEE Sensors Journal, 2012, 12(6): 2249-2261.

[2] AHN C K, SHI P, BASIN M V. Two-dimensional dissipative control and filtering for Roesser model. IEEE Trans. on Automatic Control, 2015, 60(7): $1745-1759$.

[3] SONG H F, LIU J M, LIU B, et al. Two-dimensional materials for thermal management applications. Joule, 2018, 2(3): $442-463$.

[4] YE S X, WANG W Q. Stability analysis and stabilisation for a class of 2-D nonlinear discrete systems. International Journal of Systems Science, 2011, 42(5): 839-851.

[5] HUANG S P, XIANG Z R. Delay-dependent robust H-infinity control for 2-D discrete nonlinear systems with state delays. Multidimensional Systems and Signal Processing, 2014, 25(4): $775-794$.

[6] BU X H, WANG H Q, HOU Z S, et al. Stabilisation of a class of two-dimensional nonlinear systems with intermittent measurements. IET Control Theory and Applications, 2014, 8(15): $1596-1604$.

[7] BU X H, WANG H Q, HOU Z S, et al. H $\infty$ control for a class of 2-D nonlinear systems with intermittent measurements. Applied Mathematics and Computation, 2014, 247: 651-662.

[8] KOKIL P. An improved criterion for the global asymptotic stability of 2-D discrete state-delayed systems with saturation nonlinearities. Circuits, Systems and Signal Processing, 2017, 36(6): $2209-2222$.

[9] XU H, ZOU Y, LU J, et al. Robust control for a class of uncertain nonlinear two-dimensional systems with state delays. Journal of the Franklin Institute, 2005, 342(7): 877-891.

[10] KHALIL H K. Nonlinear systems. New Jersey: Prentice Hall, 2002.

[11] DHAWAN A, KAR H. LMI-based criterion for the robust guaranteed cost control of 2-D systems described by the Fornasini-Marchesini second model. Signal Processing, 2007, 87(3): $479-488$.

[12] DHAWAN A, KAR H. An LMI approach to robust optimal guaranteed cost control of 2-D discrete systems described by the Roesser model. Signal Processing, 2010, 90(9): $2648-$ 2654.

[13] XU J M, YU L. Delay-dependent guaranteed cost control for uncertain 2-D discrete systems with state delay in the FM second model. Journal of the Franklin Institute, 2009, 346(2): $159-174$.

[14] PENG D, GUAN X P, LONG C N. Robust output feedback guaranteed cost control for 2-D uncertain state-delayed sys- 
tems. Asian Journal of Control, 2007, 9(4): 470-474.

[15] ARZEN K E. A simple event-based PID controller. Proc. of 14th IFAC World Congress, 1999, 18: $423-428$.

[16] ASTROM K J, BERNHARDSSON B. Comparison of periodic and event based sampling for first order stochastic systems. Proc. of the 14th IFAC World Congress, 1999: 301 - 306.

[17] LUNZE J, LEHMANN D. A state-feedback approach to eventbased control. Automatica, 2010, 46(1): 211-215.

[18] HEEMEL W, SANDEE J H, VAN D B. Analysis of eventdriven controllers for linear systems. International Journal of Control, 2008, 81(4): 571-590.

[19] WANG X, LEMMON M D. Event-triggering in distributed networked control systems. IEEE Trans. on Automatic Control, 2011, 56(3): 586-601.

[20] YUE D, TIAN E, HAN Q L. A delay system method for designing event-triggered controllers of networked control systems. IEEE Trans. on Automatic Control, 2013, 58(2): $475-$ 481.

[21] HU S, YIN X, ZHANG Y, et al. Event-triggered guaranteed cost control for uncertain discrete-time networked control systems with time-varying transmission delays. IET Control Theory and Applications, 2012, 6(18): 2793 - 2804.

[22] YAN H C, ZHANG H, YANG F, et al. Event-triggered asynchronous guaranteed cost control for Markov jump discretetime neural networks with distributed delay and channel fading. IEEE Trans. on Neural Networks and Learning Systems, 2018, 29(8): 3588-3598.

[23] PENG C, HAN Q L. A novel event-triggered transmission scheme and L-2 control co-design for sampled-data control systems. IEEE Trans. on Automatic Control, 2013, 58(10): $2620-2626$.

[24] PENG C, YANG T C. Event-triggered communication and $\mathrm{H} \infty$ control co-design for networked control systems. Automatica, 2013, 49(5): 1326-1332.

[25] BARMISH B R. Necessary and sufficient conditions for quadratic stabilizability of an uncertain system. Journal of Optimization Theory and Applications, 1985, 46(4): 399-408.

\section{Biographies}

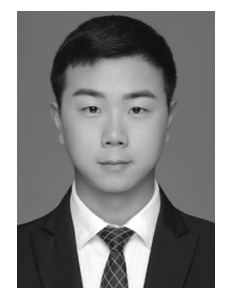

WANG Sen was born in 1994. He received his bachelor's degree in automation control from the School of Electrical Engineering and Automation, Henan Polytechnic University, Jiaozuo, China, in 2016. He is currently pursuing his master's degree in control science and engineering with the School of Electrical Engineering and Automation, Henan Polytechnic University, Jiaozuo, China. His current research interests include two-dimensional system theory, network control systems.

E-mail: wangsmmm@126.com

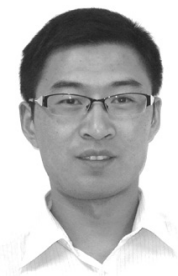

BU Xuhui was born in 1981. received his bachelor's and master's degree in automation control from Henan Polytechnic University, Jiaozuo, China, in 2004 and 2007, respectively, and Ph.D. degree in control thoery and application from Beijing Jiaotong University, Beijing, China, in 2011. He is currently an associate professor in Henan Polytechnic University, Jiaozuo, China. He has authored over 50 peerreviewed journal papers and over 20 papers in prestigious conference proceedings. His research interests include data-driven control, iterative learning control, traffic control and networked system control.

E-mail: buxuhui@gmail.com

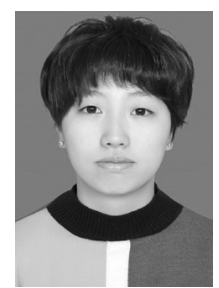

LIANG Jiaqi was born in 1992. She is currently a doctor graduate student in mining control engineering, with Henan Polytechnic University, Jiaozuo, China. She received her bachelor's degree in computer science and technology from Henan University of Science and Technology and master's degree in control science and engineering from Henan Polytechnic University, in 2014 and 2018, respectively. Her research interests include iterative learning control, mulit-agent systems control.

E-mail: 1459485470@qq.com 